\title{
Section B
}

\section{High-Energy Phenomena in and Emission from Astrophysical Plasmas}




\title{
Neutron Stars in Supernova Remnants
}

\author{
F. Pacini \\ Arcetri Astrophysical Obs. and Dept. of Astronomy \& Space Sciences, \\ University of Florence, Largo E. Fermi 5, I-50125, Firenze, Italy
}

\begin{abstract}
.
In the following, we shall briefly summarize some facts and ideas concerning the presence of neutron stars in supernova remnants. While sources similar to the Crab Nebula require the presence of a central energetic object, shell-type remnants such as Cas $\mathrm{A}$ are compatible with the presence of neutron stars releasing a weak relativistic wind.
\end{abstract}

Supernova remnants are usually classified into two extreme categories: shelltype and filled-center (plerions). In the case of shell remnants, the edges of the source appear bright, the interior rather faint. The typical radio spectrum is steep $\left(S_{\nu} \propto \nu^{\alpha}\right.$ with $\left.\alpha \simeq-0.5\right)$ and is due to synchrotron radiation from relativistic electrons produced by shocks in the region where the expanding debris interact with the circumstellar/interstellar medium. Cas A is the prototype of shell-type remnants.

On the opposite end, the Crab Nebula has been assumed to be the typical plerion, where a central neutron star continuously converts its rotational energy into a magnetized, relativistic wind. This wind expands and produces a centerfilled nebular emission which has a rather flat radio spectrum $(\alpha \simeq-0.2)$. It is not surprising that several remnants show both characteristics (internal emission and bright limbs) since some plerions expand into a relatively dense medium (composite remnants). For more details, we refer the reader to a broad review by Frail (1998) and to a full coverage of the subject in the Proceedings of the Arcetri - Elba Workshop "Relationship between Neutron Stars and Supernova Remnants" (Bandiera et al. 1998). We also refer to two accompanying papers in these Proceedings which deal with the Crab Nebula (Amato 2000) and with plerions in general (Bandiera 2000). Among the 215 catalogued supernova remnants (Green 2000), 85\% are of the shell-type.

In the past, it was widely held that plerions contain a neutron star while shell-type remnants do not, either because the explosion blows apart the entire star or because the central object becomes a black hole. The basis for this belief was the lack of evidence for an internal radio pulsar and/or for a source of relativistic wind. On the other hand, already in the early work on pulsars, it had been suggested that magnetic fields of neutron stars can reach values up to, say, $10^{14}-10^{15}$ gauss (Woltjer 1968). The initial loss of rotational energy would then be very fast and the central neutron star would soon become unable to produce a strong relativistic wind. 
In this framework, the possible presence of a neutron star hidden in Cas A (with period $P \geq 0.7 \mathrm{sec}$ and $B>10^{14}$ gauss) was discussed by Cavaliere \& Pacini (1970).

It is also worth recalling that the estimate for the rate of core-collapse supernovae (around one every 30-50 years) is about a factor of two larger than the same estimate for the birthrate of radio pulsars (about one every 100 years). Despite the uncertainty of these estimates, this suggests the existence of a large fraction of neutron stars which do not appear as radio pulsars (Helfand \& Becker 1984; Helfand 1998).

Indeed, in recent years, the observational evidence for the presence of neutron stars in supernova remnants has changed, largely because of observations in the X- and $\gamma$-ray bands. According to Frail (1998), at least 19 supernova remnants contain neutron stars which manifest themselves in different ways. Seven of them are classical radio pulsars (only one third of the total!); 3 are X-ray binaries; 2 are slow X-ray pulsars; 2 are soft gamma-ray repeaters; and 5 are radio quiet neutron stars (detected because of their thermal X-ray emission). Although this list is not updated, the picture has not changed substantially in its implications.

The reasons which lead to different manifestations of neutron stars in supernova remnants are not fully understood. However, the binary nature of the system (i.e., the role of accretion) or the initial period of the neutron star and/or the magnetic field strength are likely to play a dominant role in determining the evolution. These factors may not be independent. In particular, the initial rotation of neutron stars could be related to the strength of the coupling between the collapsing core and the stellar envelope (Tsuruta \& Cameron 1966; Pacini 1983). Strong magnetic fields would lead to slow initial rotation. Even if this coupling is not important, the wind produced by a newly born, strongly magnetized neutron star could damp the rotation immediately or shortly after the explosion. As a consequence, some young remnants could contain a slow neutron star unable to support a strong wind. The evidence (from the slowing down rate) for the presence of neutron stars with periods of several seconds and fields up to and above $10^{14}$ gauss in some remnants suggests that this occurs rather frequently. This could lead to the dominance of the stellar magnetic energy $\left(\simeq B^{2} R^{3}\right)$ over the rotational energy $\left(\simeq M R^{2} \Omega^{2}\right)$, a so-called "magnetar". Magnetic fields would then become the main energy source, which could be released, e.g., through flares. This possibility, already envisaged by Woltjer (1968), has been revived in more recent times as a possible explanation of the soft gamma-ray repeaters (Duncan \& Thompson 1992). We stress, however, that the standard determination of the field value is based upon the energy loss in a dipole field: any deviation from the dipolar geometry inside the speed of light cylinder (or, even worse, the existence of alternative slowing-down mechanisms) would invalidate the determination of the strength on the stellar surface.

In any case, it is now evident that the lack of a radio pulsar or of a plerionic component cannot be taken as proof for the nonexistence of a neutron star inside a supernova remnant. In this case, only observations can tell us whether the central object is a slow rotator with a strong magnetic field or, at the opposite end, a fast but weakly magnetized neutron star (or any other combination of rotation and field strength unable to provide a strong pulsar wind). 
Finally, we note that SN 1987A does not show, in its light curve, evidence for a pulsar energy input larger than $2 \times 10^{36} \mathrm{erg} \mathrm{s}^{-1}$ (Danziger, private comm.). This lends additional support to the idea that some remnants may contain neutron stars less energetic than those usually present in plerions.

Note added (October 1999). After I.A.U. Symposium 1995 took place, a central point source (neutron star?) in Cas A has indeed been found by the AXAF-Chandra X-ray satellite, shortly after its launch (Tananbaum et al. 1999). Observations in the various bands are currently underway. These will be able to provide more information about the nature and possible periodicity of this source, as well as about the correctness of the points discussed above.

Acknowledgments. I am indebted to E. Amato, R. Bandiera, M. Salvati, and L. Woltjer for many discussions on the subject of this talk. This work was partly supported by the Italian Space Agency.

\section{References}

Amato, E. 2000, these Proceedings

Bandiera, R. 2000, these Proceedings

Bandiera, R., Masini, E., Pacini, F., Salvati, M., \& Woltjer, L. (eds.) 1998, Mem. Soc. Astron. Italy, 69, no. 4

Cavaliere, A., \& Pacini, F. 1970, ApJ, 159, L21

Duncan, R. C., \& Thompson, C. 1992, ApJ, 392, L9

Frail, D. A. 1998, in NATO ASI Series C, 515, The Many Faces of Neutron Stars, eds. R. Buccheri, J. Van Paradijs, \& M. A. Alpar (Dordrecht: Kluwer), 179

Green, D. A. 1996, in IAU Colloq. 145, Supernovae and Supernova Remnants, eds. R. McCray \& Z.-R. Wang (Cambridge: Cambridge Univ. Press), 341

Helfand, D. J. 1998, Mem. Soc. Astron. Italy, 69, no. 4, 791

Helfand, D. J., \& Becker, R. H. 1984, Nature, 307, 215

Pacini, F. 1983, A\&A, 126, L11

Tananbaum, H. 1999, IAU Circ. 7246

Tsuruta, S., \& Cameron, A. G. W. 1966, Nature, 211, 356

Woltjer, L. 1968, ApJ, 152, L179 